\title{
Design Optimization for DNA Nanostructures
}

\author{
Jacob Girard ${ }^{1,2}$, Andrew Gilbert ${ }^{1,2}$, Daniel Lewis ${ }^{3}$, and Mary Spuches ${ }^{2,4}$ \\ Department of Mathematics \\ Saint Michael's College \\ One Winooski Park \\ Colchester, Vermont 05439 USA
}

Received: September 9, 2010

Accepted: February 26, 2011

\begin{abstract}
This paper is concerned with minimizing the cost of self-assembling DNA nanostructures by minimizing the number of different components used in the construction. We first describe the nanostructures, then give a combinatorial formalization of the assembly process and demonstrate that the octet truss provides an accurate geometric framework for current branched junction molecule assembly. We choose the octet truss because it is highly symmetric and has an appropriate number of edges for the application. We develop a method of differentiating among branched junction molecules, the basic building blocks of the nanostructures, within this structure. In the mathematical model, we represent the branched junction molecules graphically with 'tiles'. We use this approach to find the minimum number of tiles necessary to construct Platonic and Archimedean solids naturally occurring within the octet truss. This will be useful and cost efficient for the chemists and biologists who actually build these branched junction molecules because once a branched junction molecule is created, a lab can make many copies of it.
\end{abstract}

\section{INTRODUCTION}

Self-assembling nanostructure technology uses the self-replication and complementary chemical binding properties of DNA to create DNA molecules with a variety of non-linear structures such as ultrafine meshes and the skeletons of Platonic solids. This is an emerging technology with great potential and realized applications in bimolecular computing, drug delivery, and electronics $[1,16,20,23]$. A necessary step in the development of any self-assembling

1 The project described was supported by the Vermont Genetics Network through Grant Number P20 RR16462 from the INBRE Program of the National Center for Research Resources (NCRR), a component of the National Institutes of Health $(\mathrm{NIH})$. Its contents are solely the responsibility of the authors and do not necessarily represent the official views of NCRR or NIH.

2 We also have received funding from CURM, the Center for Undergraduate Research in Mathematics. CURM is funded by National Science Foundation grant DMS 0636648 and by Brigham Young University.

3 Support was also provided through NASA by the Vermont Space Grant Consortium.

4 This material is based upon work supported by the National Science Foundation, Grant No. 1001408. nanostructure is designing the component molecules. The construction method considered in this paper uses components called branched junction molecules, which consist of several "arms" or "branches" of DNA extending from a common center. The active site at the terminus of an arm is called a "cohesive end" or "sticky end" $[13,14,18]$. Several of these branched junction molecules attach to one another via basepairings at the ends of the branches to form the desired nanostructure.

We have approached the problem by developing a method of categorizing these component molecules through combinatorial representations, which are called tiles. The finalpartof this paper examines Platonic and Archimedean solids embedded in the octet truss.

\section{BIOLOGICAL BACKGROUND}

\section{a. Branched Junction Molecules}

We aim to minimize the cost of producing DNA nonconstructs by minimizing the number of component molecules 
required for their assembly. Each nanoconstruct is assembled from branched junction molecules. However, the creation of many distinct types of branched junction molecules costs time and money, thus the design for a particular structure that uses the fewest different types of molecules will likely be the most cost effective.

The nanostructures that we consider here are structurally similar to wire-frame models [7]; that is, the structures consist of several lengths of DNA, corresponding to the edges of a graph that connect at certain points, which may be thought of as vertices (see Figures 12 and 14). There are a number of approaches to inducing self assembly [22]. We focus here on the branched junction method of assembly [20]. Thus, we assume that each component molecule consists of some number of arms of DNA that extend from a central vertex and end with an active site with bonding specificity.

We are interested in branched junction molecules that follow a specific set of structural guidelines which have been determined in part as a result of present production capabilities. We assume here that the molecules have straight, rigid arms of unit length that are fixed with respect to the central vertex in two ways: first, the positions of the arms are fixed with respect to one another; second, the arms are fixed with respect to the configuration such that the active areas of the cohesive ends do not change position by twisting; that is, we are assuming that the arms do not experience twist strain, which is the compression or elongation of a DNA strand as a result of external forces [19]. Thus, DNA strands are either too short to curve or are reinforced for rigidity.

These two assumptions are based on our understanding of the way that these molecules are designed. For simplicity, our diagrams make it appear as though an arm consists of a single double helix molecule. However a design for a rigid arm may be more complex, for example, consisting of more than two strands of DNA, bonded together so that the arm itself is rigid and straight [19]. These arms begin at a central vertex and terminate with an active site, and we can discuss them as if they were the simpler versions presented in the diagrams because the added complexity does not change their combinatorial function.

We consider only those molecules with up to twelve arms, because while it is theoretically possible to create branched junction molecules with an arbitrary number of arms, twelve is the current state of the art [20]. We also require that the final DNA structure be complete, that is, it must have no unmatched cohesive ends.

We wish to design component molecules so that no complete structures smaller than the target structure may form. By a complete structure we mean that there are no unmatched arms in the target structure. A design that does not allow smaller structures to form is better than one that does because the target structure is formed with the minimum number of tiles.

We do this because if smaller structures can form them it is far more probable that they will, which means that most of the product will consist of these smaller structures. Therefore, a design that does not allow smaller structures to form is better than one that does.

\section{b. DNA Background Information}

It is important to discuss some basic properties of DNA. Deoxyribonucleic acid (DNA) is the material that many forms of like use to store genetic information. DNA is double-stranded: that is, it consists of two chains that bond together to form a double helix, which looks something like a twisted ladder [22]. Two single strands cohere as a result of bonds between notrogenous bases protruding from each strand. Each nitrogenous base is attached to a backbone of sugar and phosphate molecules which bond in a regular, repeating way to form one strand. The information in DNA is stored as a code consisting of permutations of the four nitrogenous bases within the DNA molecule: adenine $(A)$, guanine $(G)$, cytosine $(C)$, and thymine $(T)$. It is this sequence of bases in a strand of DNA that determines the information utilized by proteins to perform tasks like cell maintenance $[9,16,17]$. The nitrogenous bases in DNA bond to one another in a specific way: A bonds with $T$, and $T$ bonds with $G$, to form units called base pairs, which are the rungs of the twisted DNA ladder, In order for two strands to join, they must have complementary sequences of nitrogenous bases; that is, if 
one strand is ATCGATG then the other strand must be TAGCTAC, so that each nitrogenous base is able to bond with a base in the opposite strand (see Figure 1). A cohesive end of a branched junction molecule consists of a single-stranded fragment that extends past the doublestranded part of an arm. Self-assembly is then a result of the tendency of complementary single-strands to bond with one another, uniting half-edges to complete the structure.

Definition 1. A sticky end is an end of the DNA double-stranded helix with one strand extended longer than the other. This allows for a complementary strand to attach.

DNA is discrete, which means that it consists of distinct elements, and it is also a stable molecule. It exhibits these characteristics because it must be able to store and then maintain information accurately over time. These attributes can be made to serve other purposes. DNA can be used to build nano-scale structures and to compute the solutions of problems $[1,20]$. These uses for DNA are possible because the molecule will not break down easily or unexpectedly, and because the discrete nature of the molecule allows researchers to target parts of a strand or to design molecules that will bond in a specific way. Researchers in DNA nanotechnology and DNA computing are looking for ways to manipulate DNA so that they can create useful structures. Current research is directed at developing certain structures that could potentially be used for targeted drug delivery [15] or for developing both nanoelectronics or biochip technology. Several different nanostructures have been constructed for self-assembling DNA strands, including cubes [4], truncated octahedra [24], rigid octahedra [21], and buckyballs [12].

\section{c. Problem Summary}

Given a target geometrically realized graph, determine the minimum number of branched

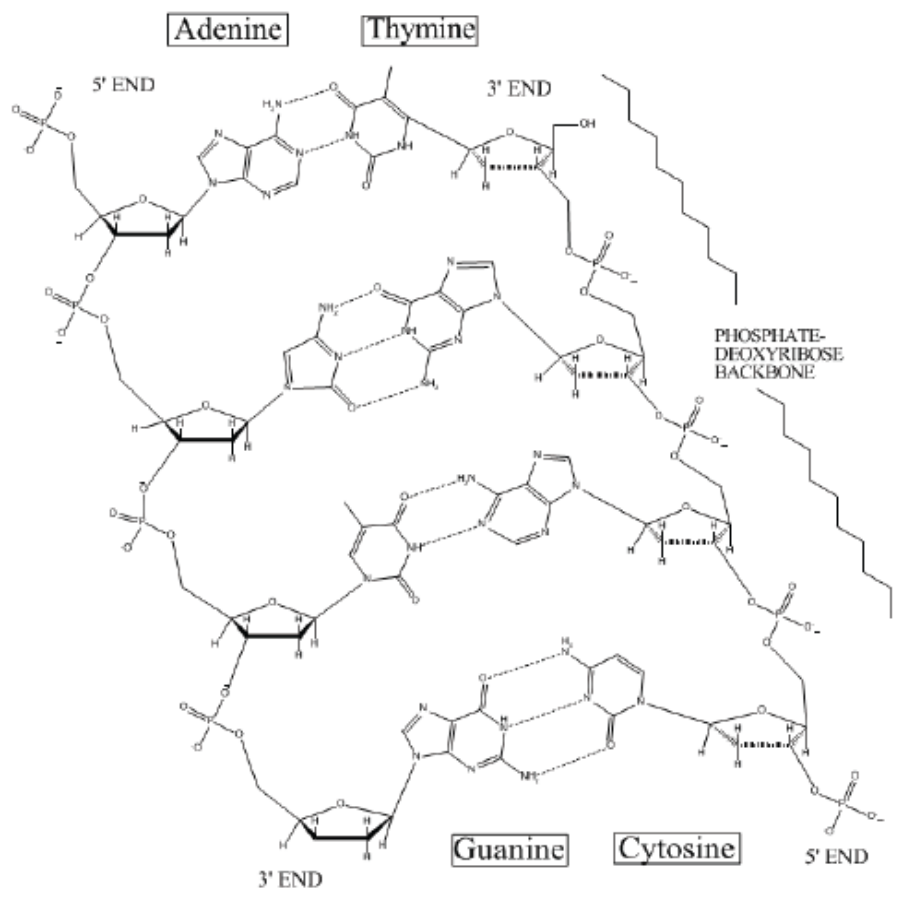

Figure 1. A depiction of double-stranded DNA. This figure shows the nitrogenous bases in DNA: adenine $(A)$, thymine $(T)$, guanine $(G)$, and cytosine $(C)$. We should notice that $A$ bonds with $T$ and that $T$ bonds with $G$. 


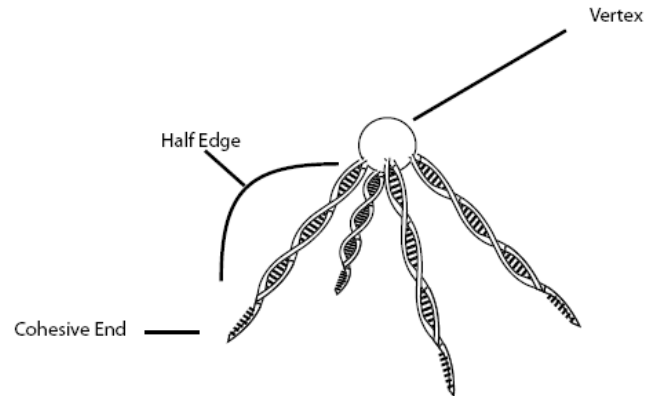

Figure 2. A depiction of the typical fourarmed tile.

junction molecules necessary to form a complex with the same structure as the target graph, subject to the following constraints:

1. Arms of branched junction molecules are straight, rigid, and of unit length.

2. The geometric positions of the arms are fixed about a vertex.

3. The arms do not experience twist strain.

4. No branched junction molecule has more than twelve arms or less that two arms.

5. Final DNA structures must be complete, that is, have no unmatched cohesive ends.

6. No design may allow structures smaller than the target structure to form.

\section{MATHEMATICAL BACKGROUND}

a. Tiles

Definition 2. A tile is a graphical representation of a branched junction molecule with cohesive or sticky ends at the end of each arm (see Figure 2). It consists of a vertex with a number of labeled halfedges in a fixed geometric arrangement about the vertex. When two arms are bonded together, they will be referred to as an edge.

Each tile has three separate
characteristics which determine its
composition. These are the configuration of
arms about the central vertex, the particular
permutation and identities of the cohesive
ends of the arms, and the attachment angles

of a particular tile with respect to those with which it may attach. Problem formulation necessitates a method of communicating and manipulating these three characteristics.

\section{b. Parity Proposition}

Cohesive ends of a branched junction molecule are encoded by cohesive end types (letter-labels on the tiles) such that a cohesive end labeled with an unhatted letter can adjoin to a cohesive end labeled with its complementary hatted label (e.g., cohesive end types $c$ and $\hat{c}$ represent complementary strands of bases and so could form a bond-edge.) A target structure is constructed fromthese branched junction molecules where the arms are joined by complementary bases on the sticky ends. For our purposed, we only wish to consider those DNA complexes which are complete, meaning they have no unmatched sticky ends. This brings us to the idea of a pot for our mathematical design.

Definition 3. A pot $P$ is a set of tile types such that for each cohesive end of type $h$ that appears in any tile $t_{i} \in P$, there exists a cohesive end of type $\hat{h}$ (its complement) in some tile $t_{j} \in P$ (possibly $I=\jmath$ ) [3].

Proposition 1. If $P$ is a pot $=\left\{t_{1} \ldots t_{n}\right\}$, and each tile $t_{j}$ has $A_{i, j}$ cohesive ends of type $a_{i}$ and $\bar{A}_{i, j}$ cohesive ends of type $\hat{a}_{i}$, then the observations below are immediate consequences of requiring complexes to be complete.

a) A graph $G$ with $n$ vertices may be realized by the pot $P$ only if there are non-negative integers $r_{j}$ for $j=$ $1 . . . p$ (representing the number of times each tile of type $t_{j}$ is used in the construction of $G)$ with $\sum_{j} r_{j}=n$ and such that $\sum_{j} r_{j} A_{i, j}=\sum_{j} r_{j} \hat{A}_{i, j}$ for all $i$. That is, the number of hatted sticky ends of each type used in the construction of $G$ must equal the number of cohesive sticky ends of 


\section{Pot P:}
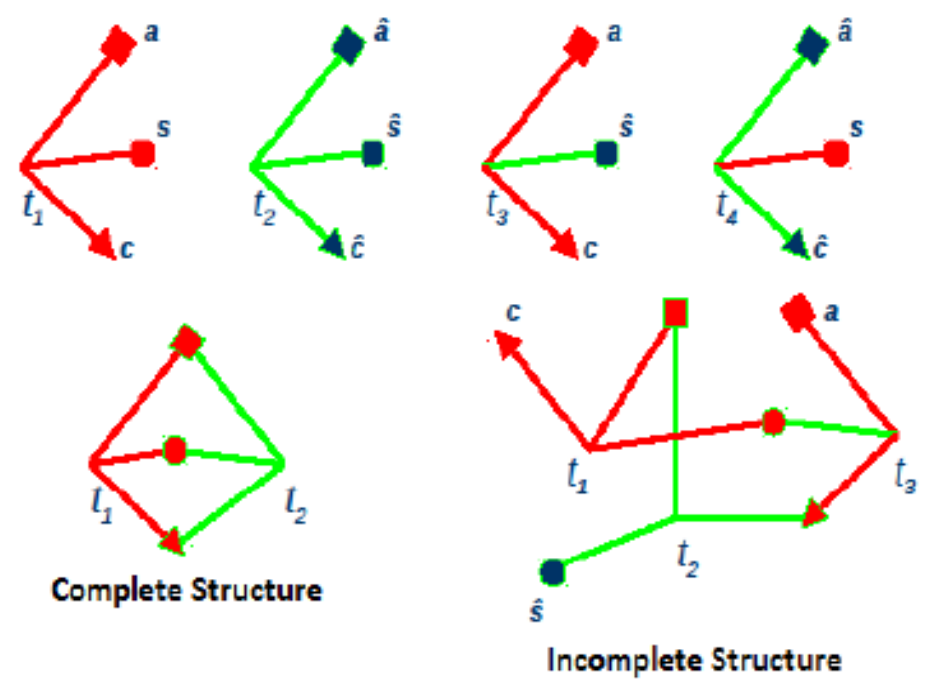

Figure 3. An example of a pot of tiles, $P$, which can form both complete and incomplete structures. The bottom left figure is made up of tile 1 and tile 2 from the pot $P$. It is complete because the three arms of tile 1 have complementary arms found on tile 2 . In particular, $a$ bonds with $\hat{a}, c$ bonds with $\hat{c}$, and $s$ bonds with $\hat{s}$. The bottom right figure is incomplete because is has three extra arms that do not have a complementary sticky end. Namely the cohesive ends $a, c$, and $\hat{s}$ are left unmatched. If tile 4 is attached to this figure, a complete structure is formed.

the same type that appear in the construction.

b) The total number of hatted cohesive end types must equal the total number of unhatted cohesive end types in a complete complex. While trivial, this observation will be equal in parity arguments.

\section{c. The Octet Truss}

The geometric attributes of our design prompt us to turn to the octet truss of R. Buckminster Fuller [10]. We will consider structures in the octet truss that are composed of vertices and edges running between these vertices. The octet truss consists of regular tetrahedra and octahedra, and it is the only way to tile three-space in a semi-regular way (see Figure 4). Note that the angle formed by any two edges must be $\pi / 3, \pi / 2,2 \pi / 3$, or $\pi$ radians. The $\pi / 2$ right angles are created by two edges incident to the same vertex on the equator of an octahedon [6].

The design constraints outlined in Section II c lead us to consider structures in the octet truss. The octet truss is ideal because no vertex in the octet truss can ever have more than twelve arms, because all the edges are rigid and of unit length, the edges are distributed relatively evenly about the center vertex, and because the octet truss is highly symmetric.

\section{d. Configuration}

We will begin by developing a system of categorizing tiles by the geometric configuration of their atoms. These configurations around the different tiles in a target structure are forced, that is, if a tile has a specific target complex embedded in the octet truss, each tile's configuration is determined. Otherwise, the structure would not be completely determined.

The half-edges around any vertex in the octet truss may be divided into sets by grouping them such that all the half-edges in a particular set are in the same plane. Careful inspection will show that it is impossible to find just two planes which contain all the half-edges; therefore, it is necessary to use at least three planes. Another important point is that, considering the sets of arms in particular planes, if one crosses two planes at random, it is often true that the sets are not disjoint. To 


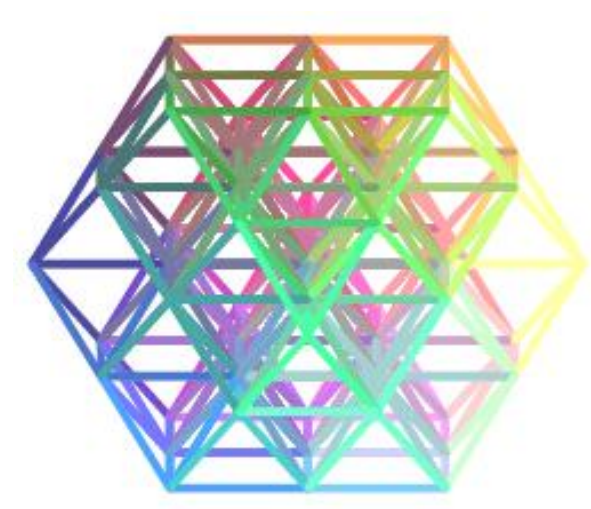

Figure 4. A subset of the octet truss. There are only four angles that will be formed by any two edges in this figure: $60^{\circ}, 90^{\circ}, 120^{\circ}$, and $180^{\circ}$.

Avoid naming half-edges in a way that might lead to having more than one name for any one half-edge, we must choose the three planes containing four half-edges, with each arm orthogonal to the half-edges immediately to its left and right. Then we may name the planes with the Greek letters $\alpha, \beta$, and $\gamma$, and give each arm in each plane a number between one and four, inclusive. The outcome is that each half-edge has a label consisting of a Greek letter and then a number. To number the vertices, we choose a half-edge and label it $\alpha_{1}$. The $\alpha$-plane is then labeled arm by arm with increasing numbers in a clockwise direction when looking at it from the top. We define up to be the direction of any vector perpendicular to the $\alpha$-plane and away from the region in space created by the $\alpha$-plane and containing the $\beta_{1}$ and $y_{1}$ half-edges. These two halfedges are the two closest to $\alpha_{1}$ and below the $\alpha$-plane. $\beta_{1}$ is closer to $\alpha_{4}$ than $\gamma_{1}$. The $\beta$ and $Y$-planes are then labeled with increasing numbers such that $\beta_{2}$ and $\gamma_{2}$ are above the $\alpha$-plane (see Figure 7 ).

We refer to this construction as a labeling cage. To name a configuration, we place it in the labeling cage so that the arms are in the lexicographically minimal position (see Figures 7 and 8). Based on a tile's lexicographical labeling, we classify the tiles. We now find all the configurations of two and three arms about a vertex so that we can know what possibilities there are when we are trying to minimize the number of tiles needed to build a DNA structure.

\section{e. The Problem of Orientation}

Fixing the geometry of the arms about the central vertex of a tile means that the cohesive end of the arm is oriented in a certain way in the branched junction molecule being represented. Any fixed arm that bonds to it will be oriented in a particular way, resulting in the two tiles being oriented in only one way with respect to one another.

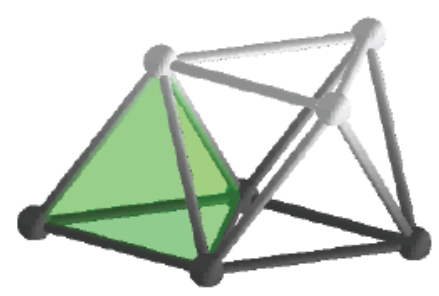

Figure 5. The basic unit of the octet truss. The left, shaded area of the figure is a tetrahedron and the right side of the figure forms an octahedron. This picture illustrates some of the symmetry found in the octet truss.

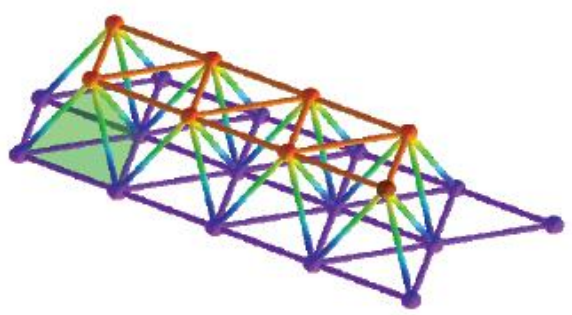

Figure 6. A horizontal layer of the octet truss. This figure continues infinitely, repeating the tetrahedron and octahedron shapes in all directions.

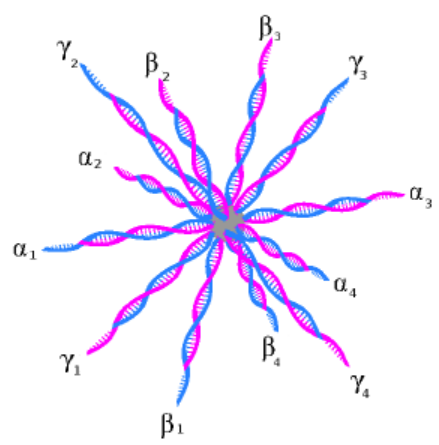

Figure 7. This figure represents our labeling convention for all twelve arms of a single vertex in the octet truss. We denote the arms with Greek letters and numerical subscripts: $\alpha_{1}, \ldots, \alpha_{4}, \beta_{1}, \ldots, \beta_{4}, \bigvee_{1}, \ldots, \bigvee_{4}$. 


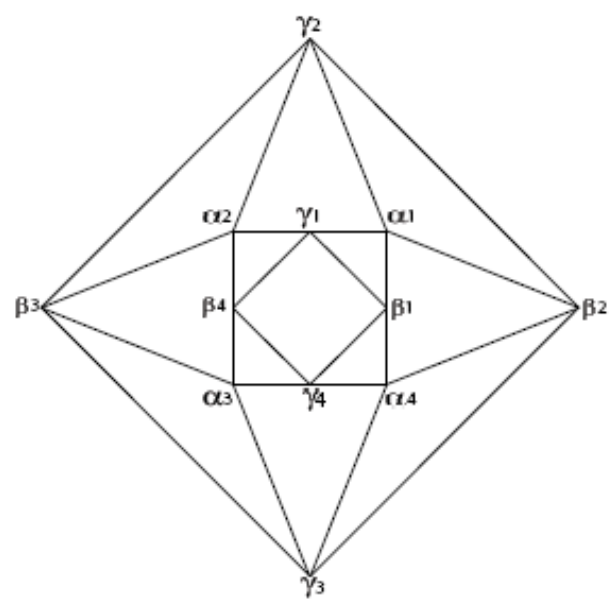

Figure 8. This is a schlegel diagram of the cuboctahedron formed by the vertices adjacent to a single vertex in the octet truss and the edges between these vertices. The labels correspond to the endpoints of the arms in Figure 6.

This presents a problem because, even if two tiles have the same configuration and cohesive ends, they may bond to a third tile type along the same bond-edge in different ways such that different orientations result (see Figure 9). The two tiles would therefore be different tiles simply because their arms are fixed in different ways, resulting in molecules with different geometries. In each of our constructs we must check the way tiles are oriented in respect to one another.

In order to address orientation, we check any construction of a target structure to make sure that the design is possible. We number the arms of every tile in the pot for a particular structure and make sure that any bond using the same arms always orients the two tiles in exactly the same way. We define the bond angle formally below.

Definition 4. Let $\mathrm{T}_{1}$ and $\mathrm{T}_{2}$ be two different tiles found in the octet truss, written in lexicographical minimal order, and let $\varepsilon_{1}, \varepsilon_{2}$ $\in\left\{\alpha_{1}, \ldots, \alpha_{4}, \beta_{1}, \ldots, \beta_{4}, \gamma_{1}, \ldots, \gamma_{4}\right\}$ with $\varepsilon_{1}$ an arm of $T_{1}$ and $\varepsilon_{2}$ an arm of $T_{2}$. Further suppose that $\varepsilon_{1}$ and $\varepsilon_{2}$ have complementary sticky ends so that the tile $T_{1}$ and $T_{2}$ may adjoin. Now let $\sigma_{i}$ be the lexicographical minimal arm of $T_{i}$ omitting $\varepsilon_{i}$ and its antipodal arm (antipodal meaning that the two edges do not form a $180^{\circ}$ angle between them). Write
$P\left(\varepsilon_{\mathrm{i}}, \sigma_{\mathrm{i}}\right)$ for the plane through $\varepsilon_{\mathrm{i}}$ and $\sigma_{\mathrm{i}}$. Then the bond angle between $\left(T_{1}, \varepsilon_{1}\right)$ and $\left(T_{2}, \varepsilon_{2}\right)$ is the angle formed between the two planes $P\left(\varepsilon_{1}, \sigma_{1}\right)$ and $P\left(\varepsilon_{2}, \sigma_{2}\right)$.

Definition 5. Two branched junction molecules are called similar if their arms have the same configuration and cohesion end types.

Definition 6. Two branched junction molecules are called equivalent if they are similar and if their bond angles with any other tiles are identical.

\section{f. Geometric Configuration of Tiles}

Theorem 1. There are four unique twoarmed configurations within the constraint of the octet truss.

Proof. Two half-edges and a central vertex form an angle. There are four different angles $(\pi / 3, \pi / 2,2 \pi / 3$, and $\pi)$ that can be formed by two arms incident to the same vertex. Therefore, there are exactly four unique two-edge configurations.

Our main tool for proving that we have all the three-armed configurations will be the orbits and stabilizers theorem for the symmetry group of a geometric object. The orbit of a tile is the set of positions it is moved to by the symmetry group, i.e. the other locations in the cuboctaheron where it "fits". The stabilizer of a tile is the subgroup of the symmetry group that fixes the tile. The product of the sizes of the orbit and stabilizer is the size of the symmetry group [11].

The symmetry group of the cuboctahedron is the chiral symmetry group of the cube and the octahedron, and is or order twenty-four [5]. There are the rotations of order four about the center of each face, the rotations of order three about the center of each vertex, and the rotations of order two about the line from the midpoint of an edge to the midpoint of its antipodal edge.

We must first contend with the problem of the $2 \pi / 3$ angle.

Lemma 1. If a planar configuration contains an angle of $2 \pi / 3$, then there are in fact two identical configurations that must be considered when considering the orbits and stabilizers of these configurations. 


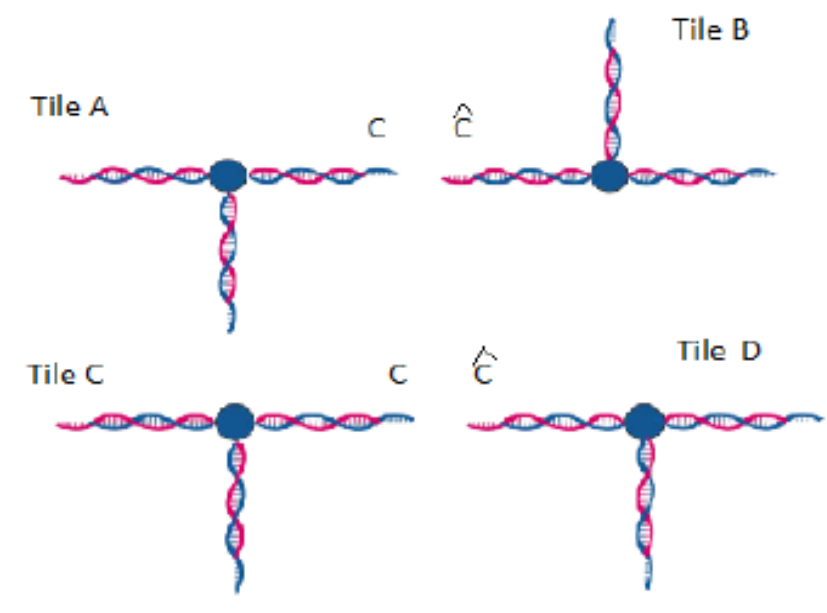

Figure 9. This figure illustrates four similar tiles. However, the bond angle between tiles $A$ and $B$ is different than the bond angle between tiles $C$ and $D$. This becomes important when creating final structures.

Proof. Consider removing a cuboctahedron with edges of unit length from the octet truss. At its center is a vertex which is connected to every other vertex that we removed (see Figure 8). The outer vertex connected to the $\alpha_{1}$ arm of the central vertex is at the corners of two triangular faces of the cuboctahedron. The only element of the chiral symmetry group which moves these triangles such that their position are exchanged is a rotation by $\pi$ radians about the axis through the central vertex and its $\alpha_{1}$ arm. There is no group element that can reverse the corners of the triangles that are away from the $\alpha_{1}$ half-edge while keeping the triangles on their respective sides of the $\alpha_{1}$ half-edge. Notice that an angle of $2 \pi / 3$ is created in selecting the central vertex and the half-edges that point toward two corners of the triangles so that the $\alpha_{1}$ arm is not selected, thus the two selected arms are not in the same categorization plane, This angle cannot be rotated onto every other angle around the central vertex because the triangles cannot be flipped, as mentioned above. From the point of view of orbits and stabilizers, any planar configuration with a $2 \pi / 3$ angle in it must actually be considered twice because it appears twice, even though it is exactly the same configuration. See Figure 10 for a depiction of this proof. Theorem 2. There are ten unique geometric configurations comprising three half-edges and a vertex of the octet truss. (See Table 2.)

Proof. There are seven configurations that have only the identity as a stabilizer $(1,2,3,4,6$, 8 ; see Table 3$)$, so each of those has configurations has an orbit of order twenty-four. There are three configurations which have the identity and a rotation by $\pi$ radians as stabilizers $(5,10$, and the necessary duplicate of 100; hence, each of those has an orbit of order twelve. One configuration has three stabilizers and an orbit of order 8 (7). Finally, there are two configurations with six stabilizers each ( 9 , and the necessary duplicate of 9); thus, they each have an orbit of order four. The sum of the orders of the orbits equal 220 , which is precisely

$$
\left(\begin{array}{c}
12 \\
3
\end{array}\right)
$$

or the number of way to choose three different edges about a central vertex from twelve total edges. There are thirteen different configurations listed above, but three of these configurations are identical to one other member of the set of configurations, as discussed in Lemma 1. Therefore, there are ten unique configurations of three edges and a central vertex inside the octet truss. $\square$ Determining the possible configurations in a like manner for 4 through 12 arms is a topic of future work. 


\begin{tabular}{|c|c|c|c|}
\hline arm & $\mathbf{x}$-coordinate & $\mathbf{y}$-coordinate & $\mathbf{z}$-coordinate \\
\hline$\alpha_{1}$ & 0 & 1 & 0 \\
\hline$\alpha_{2}$ & -1 & 0 & 0 \\
\hline$\alpha_{3}$ & 0 & -1 & 0 \\
\hline$\alpha_{4}$ & 1 & 0 & 0 \\
\hline$\beta_{1}$ & $-1 / 2$ & $1 / 2$ & $-1 / \sqrt{2}$ \\
\hline$\beta_{2}$ & $-1 / 2$ & $1 / 2$ & $1 / \sqrt{2}$ \\
\hline$\beta_{3}$ & $1 / 2$ & $-1 / 2$ & $-1 / \sqrt{2}$ \\
\hline$\beta_{4}$ & $1 / 2$ & $-1 / 2$ & $-1 / \sqrt{2}$ \\
\hline$\gamma_{1}$ & $1 / 2$ & $1 / 2$ & $1 / \sqrt{2}$ \\
\hline$\gamma_{2}$ & $1 / 2$ & $-1 / 2$ & $1 / \sqrt{2}$ \\
\hline$\gamma_{3}$ & $-1 / 2$ & $-1 / 2$ & $-1 / \sqrt{2}$ \\
\hline$\gamma_{4}$ & $-1 / 2$ & & \\
\hline
\end{tabular}

Table 1. Coordinates of Arms in the Octet Truss

\begin{tabular}{|l|l|}
\hline 1. & $\alpha_{2} \beta_{1}$ \\
\hline 2. & $\alpha_{2} \beta_{2}$ \\
\hline 3. & $\alpha_{4} \beta_{1}$ \\
\hline 4. & $\alpha_{4} \beta_{3}$ \\
\hline 5. & $\alpha_{2} \alpha_{3}$ \\
\hline 6. & $\alpha_{3} \beta_{1}$ \\
\hline 7. & $\beta_{1} \gamma_{1}$ \\
\hline 8. & $\beta_{3} \gamma_{3}$ \\
\hline 9. & $\beta_{3} \gamma_{4}$ \\
\hline 10. & $\beta_{1} \gamma_{2}$ \\
\hline
\end{tabular}

Table 2. There are ten possible unique configurations of three half-edges incident to a single vertex in the octet truss. Note that the half-edge $\alpha_{1}$ is assumed to be one of the edges in each of the configurations.

\section{g. Mathematical Design Constraints}

We identify Platonic and Archimedean solids embedded in the octet truss to determine structures that can be made with just one or two tile types. We give the minimum number of tile types required to build these structures subject to the following design constraints:

1. Tiles conform to the geometry of the octet truss as follows:

a) Arms are of unit length. b) Tiles have between 2 and 12 arms.

c) Arms are straight, rigid, and in a fixed position about the vertex.

d) Angles between the arms are either $\pi / 3, \pi / 2,2 \pi / 3$, or $\pi$ radians.

2. At the end of each arm is a letter which will only attach to its complementary end (i.e., $c$ and $\hat{c}$ are complimentary arms).

3. Final structures must be complete, that is the figures cannot have any unmatched arms.

Determining the possible configurations in a like manner for 4 through 12 arms is a topic of future work.

h. Mathematical Design Constraints

We identify Platonic and Archimedean solids embedded in the octet truss to determine structures that can be made with just one or two tile types. We give the minimum number of tile types required to build these structures subject to the following design constraints:

1. Tiles conform to the geometry of the octet truss as follows:

a) Arms are of unit length.

b) Tiles have between 2 and 12 arms.

c) Arms are straight, rigid, and in a fixed position about the vertex.

d) Angles between the arms are either $\pi / 3, \pi / 2,2 \pi / 3$, or $\pi$ radians. 


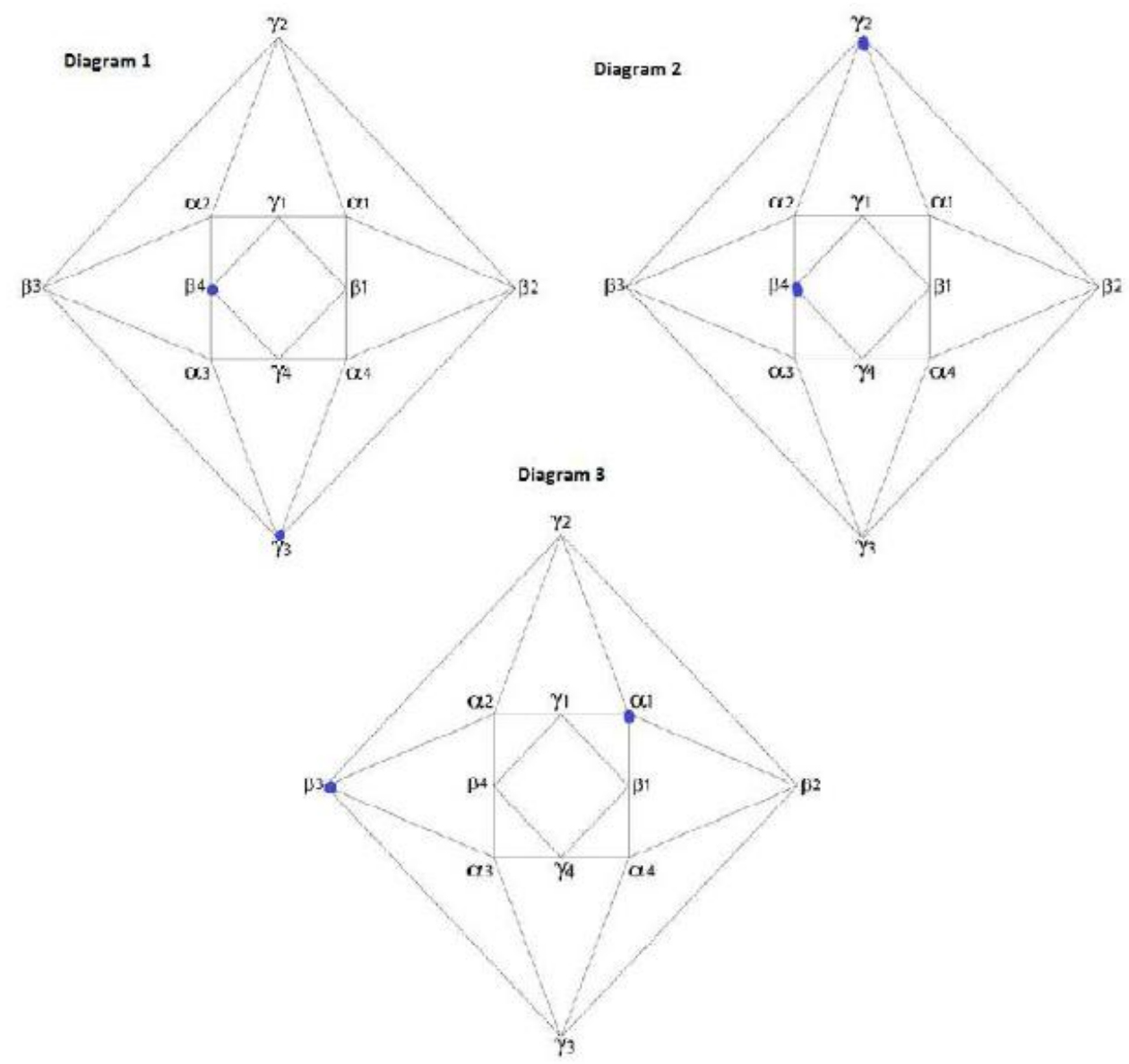

Figure 10. in Diagram 1, arms $\beta_{1}$ and $\gamma_{3}$ are removed from the Schelgal diagram. In Diagram 2, arms $\beta_{4}$ and $\gamma_{2}$ are removed. Diagram 3 depicts a tile comprised of arms $\alpha_{1}$ and $\beta_{3}$ which can rotate to fit either Diagram 1 and 2 . This occurs because there is an angle of $2 \pi / 3$ between each of these arms.

2. At the end of each arm is a letter which will only attach to its complementary end (i.e., $c$ and $\hat{c}$ are complimentary arms).

3. Final structures must be complete, that is the figures cannot have any unmatched arms.

4. No design may allow structures smaller than the target structure to form.

\section{SOME PLATONIC AND ARCHIMEDEAN SOLIDS}

We will now find provably optimal strategies for all of the Platonic and Archimedean solids that naturally occur in the octet truss: the tetrahedron, the octahedron, the truncated tetrahedron, the truncated octahedron, and the cuboctahedron. These solids are contained as subgroups within the octet truss. Thus, they automatically satisfy the design constraints in Section II c, and are promising candidates for DNA self-assembly. Indeed the cube [4], the octahedron [24], and the truncated octahedron [21] have already been built, albeit using other assembly methods. However, buckyballs use "dendtrite" structures that are essentially three-armed branched junction molecules [12]. The remaining Platonic and Archimedean solids can only be realized as homeomorphic copies in the octet truss, and we leave this for future work.

\section{a. Tetrahedron}

Theorem 3. A tetrahedron may be constructed with a minimum of two tile types. 


\begin{tabular}{|c|c|c|c|c|c|}
\hline \multicolumn{6}{|c|}{ Tetrahedron: 2 tiles required } \\
\hline & e Ty & & & Typ & \\
\hline$\alpha_{1}$ & $\beta_{1}$ & $\mathrm{~V}_{1}$ & $\alpha_{1}$ & $\beta_{1}$ & $V_{1}$ \\
\hline$\widehat{b}$ & $\hat{b}$ & $\widehat{b}$ & $a$ & $\hat{a}$ & $b$ \\
\hline
\end{tabular}

\begin{tabular}{|cccc|}
\hline Octahedron: 1 tile required & \multicolumn{3}{c|}{} \\
& Tile Type Used in Construction \\
\hline$\alpha_{1}$ & $\alpha_{4}$ & $\beta_{1}$ & $\beta_{2}$ \\
$a$ & $a$ & $\hat{a}$ & $\hat{a}$ \\
\hline
\end{tabular}

Cuboctahedron: 1 tile required

Tile Type Used in Construction

\begin{tabular}{cccc|}
\hline$\alpha_{1}$ & $\alpha_{4}$ & $\beta_{1}$ & $\beta_{4}$ \\
$a$ & $\hat{a}$ & $a$ & $\hat{a}$ \\
\hline
\end{tabular}

\begin{tabular}{|ccc|ccc|}
\hline \multicolumn{7}{|c|}{ Truncated Tetrahedron: 2 tiles required } \\
Tile Types Used in Construction \\
\hline Tile Type 1 & $\gamma_{3}$ & $\alpha_{1}$ & $\beta_{1}$ & $\gamma_{3}$ \\
$\alpha_{1}$ & $\beta_{1}$ & $\hat{a}$ & $\hat{b}$ & $a$ & $\hat{a}$ \\
$b$ & $a$ & &
\end{tabular}

Truncated Octahedron: 2 tiles required

Tile Types Used in Construction

\begin{tabular}{|ccc|ccc|}
\hline & Tile Type 1 & & \multicolumn{3}{c|}{ Tile Type 2 } \\
$\alpha_{1}$ & $\alpha_{4}$ & $\beta_{3}$ & $\alpha_{1}$ & $\alpha_{4}$ & $\beta_{3}$ \\
$b$ & $a$ & $\hat{a}$ & $\hat{b}$ & $a$ & $\hat{a}$ \\
\hline
\end{tabular}

Table 4. A summary of Results for Platonic and Archimedean Solids. Note: the Greek letters represent the arm positions and the hatted and unhatted letters represent cohesive end types.

Proof. The tetrahedron is made up of four vertices, six edges, and four equilateral triangles for faces. It requires four tiles with three arms each. By Proposition 1, the tetrahedron requires at least two tile types for its construction because the vertices are of degree three. We now present a construction that requires precisely two tile types, and does not permit the residual construction of smaller complete constructs.

Both tile types have the geometric configuration of $\alpha_{1} \beta_{1} \gamma_{1}$.

Tile Type One consists of three cohesive ends of type $\widehat{b}$ (see Figure 11).
Tile Type Two consists of one cohesive end of type $a$, one of type $\hat{a}$, and one of type $b$ (see Figure 11).

We have shown a construction which demonstrates that the absolute minimum we began with is achieveable under our present constraints (see Figure 13). We therefore have proven that the minimum number of tile types required to construct the tetrahedron is two.

b. Octahedron

Theorem 4. An octahedron may be constructed with a minimum of one tile type. 
Tile 1

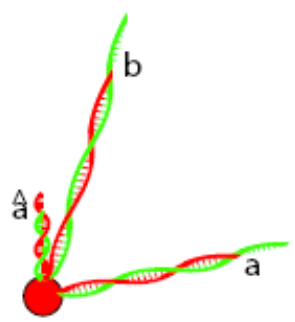

Tile 2

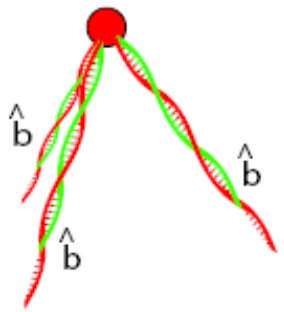

Figure 11. The two different tile types used in construction of the tetrahedron. They have the same geometric configuration, but have different cohesive ends.

Tetrahedron Assembly

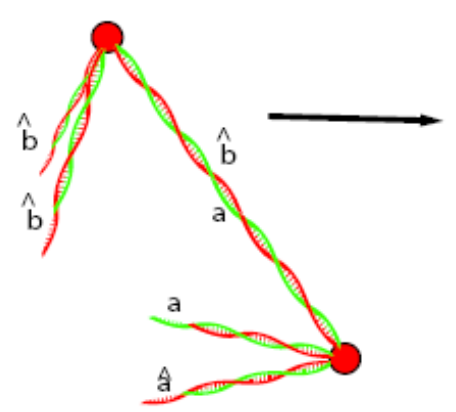

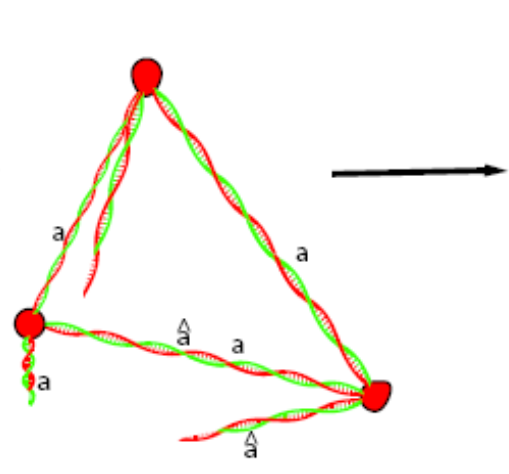

Tetrahedron

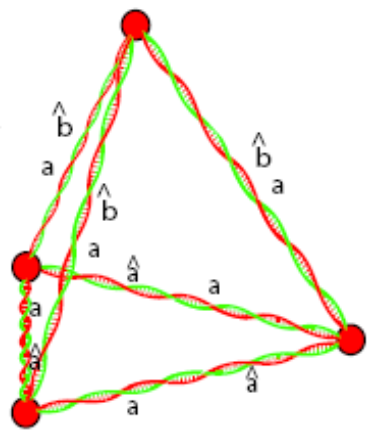

Figure 12. This is a depiction of a way that the tetrahedron might assemble from the four tiles used in this construction. Note that while the order of assembly might differ from tetrahedron to tetrahedron, the final structure is always the same.

\section{Arm Possible \\ Complementary \\ Bond Arms}

Tile 1 \& 2 Configuration: $\alpha_{1} \beta_{1} Y_{1}$

\begin{tabular}{|l|l|}
\hline 1 & 4 \\
2 & 4 \\
3 & 4 \\
4 & $1,2,3$ \\
5 & 6 \\
6 & 5 \\
\hline
\end{tabular}
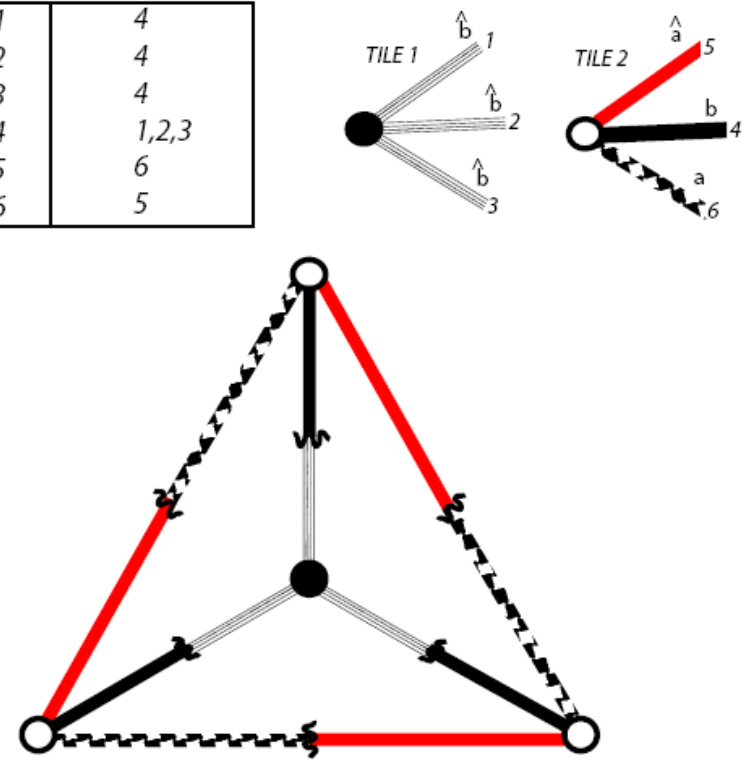

Figure 13. This diagram illustrates the construction part of the proof for the tetrahedron. 
Proof. The octahedron is made up of six vertices, twelve edges, and the faces are eight equilateral triangles. It requires six tiles with four arms each. By Proposition 1, the octahedron requires at least one tile type for its construction because the vertices are of degree four. We now present a construction that requires only one tile type.

The Tile Type for the Octahedron has the configuration $\alpha_{1} \alpha_{4} \beta_{1} \beta_{2}$, two cohesive ends of type $\hat{a}$, and two cohesive ends of type $a$ such that the two $\alpha$-ends have the same cohesive end type and the two $\beta$-ends have the same cohesive end type. Furthermore, this tile is symmetric with respect to arm orientation so that the two $a$-arms are equivalent in every way. The same is true for the $\hat{a}$-arms.

Because there is only one tile type, every tile must bond to another copy of itself. For any arm, there are two different arms that it can bond to on another tile. However, each bond will orient the two involved tiles in exactly the same way because the tile is symmetric. This means that any six tiles of this type will form an octahedron (see Figures 15). Notice that attachment angles make it impossible to form smaller or larger complete structures from these tiles.

We have shown a construction which demonstrates that the absolute minimum we began with is achieveable under our present constraints (see Figure 15). We therefore have proven that the minimum number of tiles required to construct the octahedron is one.

\section{c. Cuboctahedron}

Theorem 5. A cuboctahedron may be constructed with a minimum of one tile type.

Proof. The cuboctahedron has twelve vertices, twenty-four edges, and the faces are eight equilateral triangles and six squares. It requires twelve tiles with four arms each. By Proposition 1, the cuboctahedron requires at least one tile type for its construction because its vertices are of degree four. We now present a construction that requires only one tile type.
The Tile Type for the Cuboctahedron has the configuration $\alpha_{1} \alpha_{2} \beta_{1} \beta_{4}$, the two cohesive ends of type $\hat{a}$, and two cohesive ends of type $a$ such that the two $\alpha$-ends have different cohesive end types and the two $\beta$-ends have different cohesive end types (see Figure 16). The two $a$-arms also have the same attachment angle with any arm and hatted arm and vice versa. The $\hat{a}$-arms also have the same arm orientation. Any $a$ to $\hat{a}$ bond therefore orients its involved tiles so that their positions with respect to one another are congruent to the positions of the tiles in every equivalent bond.

Because there is only one tile type, every tile must bond to another copy of itself, For any arm, there are two different arms that it can bond to on another tile. However, each equivalent bond will orient the two involved tiles in exactly the same way as noted above. Notice that it makes a difference where the $a$ and $\hat{a}$ are located: that is, if we think of a bond as a vector directed along the bond edge from $a$ to $\hat{a}$ then the positions of involved tiles depend on which way the bond is pointing in space. Also notice that the tile has rotational symmetry of $\pi$ radians about the axis passing through its central vertex and orthogonal to the plane formed by the four endpoints of its arms. This means that even though there are many ways that these tiles could assemble to form a cuboctahedron, any number of tiles in the final structure could be removed, rotated about it axis of symmetry, and then replaced without changing the overall structure. This means that the overall structure of the cuboctahedron must form from these tiles because any combination of tiles within the restraint forms a cuboctahedron. This means that any twelve tiles of this type will form a cuboctahedron (see Figure 17). Notice that it is impossible to form any other complete structures from these tiles.

We have shown a construction which demonstrates that the absolute minimum we began with is achieveable under our present constraints. We therefore have proven that the minimum number of tiles required to construct the cuboctahedron is one. 


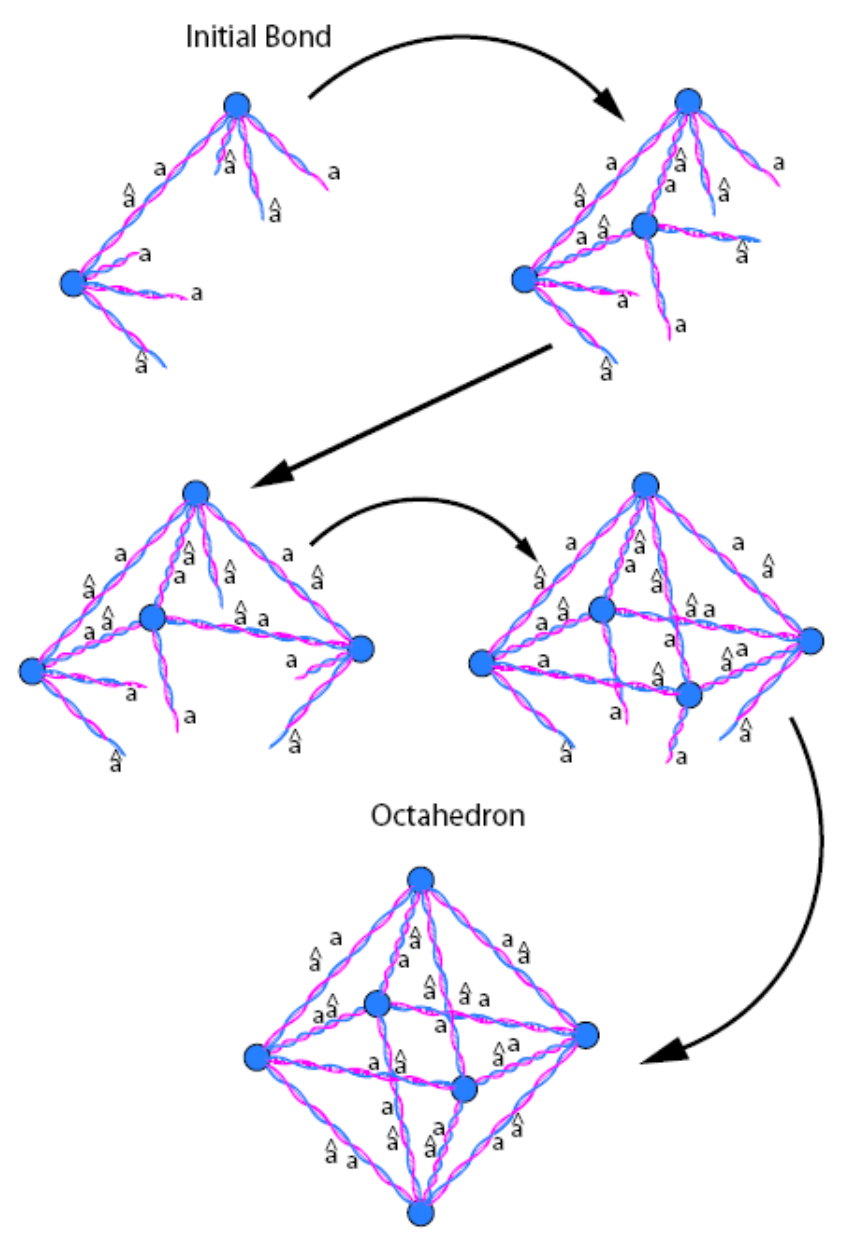

Figure 14. The construction of the octahedron. We start with one four-armed tile consisting of $a$ and $\hat{a}$ cohesive ends. Then another tile of the same configuration is bonded to the first tile. This process of adding tiles continues until the structure is complete, that is, all cohesive ends have a complementary arm attached. The octahedron takes eight four-armed tiles to be a closed and complete structure.

Remark. Notice that the tiles use more than one cohesive end type. We do this because it restricts the bonding such that there will ultimately be fewer ways for waste structures to form. The truncated tetrahedron naturally has two different types of edges. Edges of the first type form the triangle faces, while edges of the second type do not border a triangular face. So, it makes sense to use two separate cohesion end types because arms that make up the two sorts of edges never intermix. Alternatively, one may look at the tiles themselves. The $a$ and $\hat{a}$ are arms are $\pi / 3$ apart, and the third arm of each tile is $2 \pi / 3$ radians away from the other two half-edges. The truncated tetrahedron is constructed such that the arms that are $2 \pi / 3$ radians away from the other two half-edges, that is, the $b$ and $\hat{b}$ arms of any tile, always bond to one another. If the cohesive end types were all $a$ and $\hat{a}$, then undesirable bonds could form that would lead to the incidental creation of unwanted complexes.

Six tiles of Type One and six tiles of Type Two assemble as shown in Figure 18. There are twelve similar bonds composed of $a$ and $\hat{a}$ arms, and these bonds are equivalent because they orient their members in the same way. Furthermore, 
Arms Possible Complementary Bond Arms
Tile Configuration: $\alpha_{1} \alpha_{4} \beta_{1} \beta_{2}$

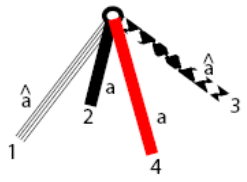

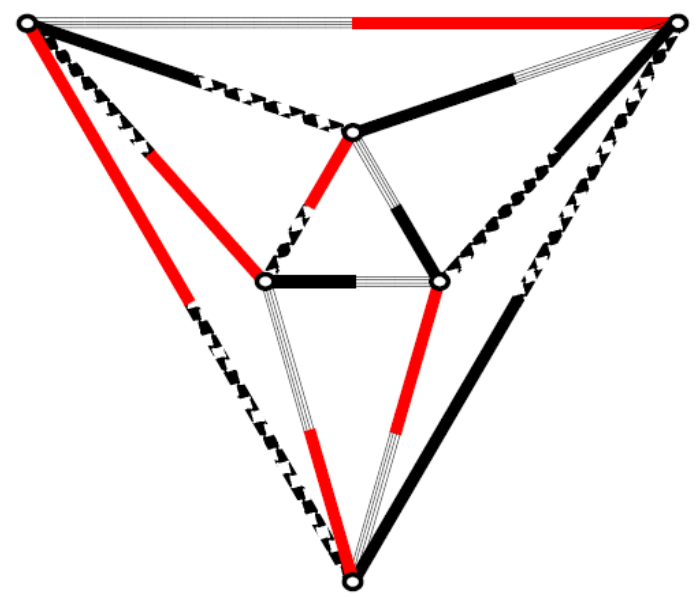

Figure 15. This diagram illustrates the construction part of the proof for the octahedron.

there are six similar bonds composed of $b$ and $\hat{b}$ arms which are equivalent because they orient their member tiles in an identical way (see Figure 18). Arm orientation does not present a problem because there are only two types of bonds and they use different cohesive end types. Note that it is impossible to form larger or smaller complete structures from these tiles.

We have shown a construction which demonstrates that the absolute minimum we began with is acheiveable under our present constraints. We therefore have proven that the minimum number of tiles required to construct the truncated tetrahedron is two.

\section{d. Truncated Octahedron}

Theorem 7. A truncated octahedron may be constructed with a minimum of two tile types.

Proof. The truncated octahedron is an Archimedean solid composed of twenty-four vertices, thirty-six edges, and six squares and eight regular hexagon faces. It requires twenty-four tiles with three arms each. By Proposition 1, the truncated octahedron requires at least two tile types for its construction because the vertices are of degree three. We now present a construction that requires precisely two tile types. Both tile types have the configuration $\alpha_{1} \alpha_{4} \beta_{3}$.

Tile Type One consists of one cohesive end of type $b$, one of type $a$, and one of type $\hat{a}$ such that the $a$ and $\hat{a}$ cohesive ends are $\pi / 2$ apart (see Figure 19).

Tile Type Two consists of one cohesive end of type $\hat{b}$, one of type $a$, and one of type and $\hat{a}$ such that the $a$ and $\hat{a}$ cohesive ends are $\pi / 2$ apart (see Figure 19).

Remark: Notice that the tiles utilize more than one cohesive end type. We do this for the same reason that we used two cohesive end types for the truncated tetrahedron. The truncated octahedron also has two different sorts of edges. Edges of the first sort form the square faces, while edges of the second sort do not border a square face. So, it makes sense to use two separate cohesive types because arms that make up the two sorts of edges never intermix. Using two cohesive end types decreases the chance that undesirable bonds will form.

Twelve tiles of Type One and twelve tiles of Type Two assemble as shown in Figure 19. There are twenty-four similar bonds 


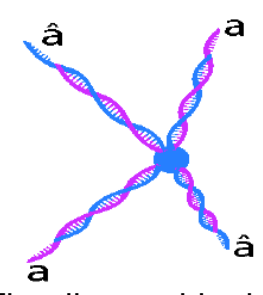

Figure 16. The tile used in the construction of the cuboctahedron. The configuration: $\alpha_{1}$ $\alpha_{2} \beta_{1} \beta_{2}$.

composed of $a$ and $\hat{a}$ arms, and these bonds are equivalent because they orient their members in the same way. Furthermore, there are twelve similar bonds composed of $\vec{b}$ and $\hat{b}$ arms which are equivalent because they orient their member tiles in an identical way (see Figure 19). Notice that it is impossible to form larger or smaller structures from these tiles.

We have shown a construction which demonstrates that the absolute minimum we began with is achieveable under our present constraints. We therefore have proven that the minimum number of tiles required to construct the truncated octahedron is two.

\section{CONCLUSIONS}

This paper is a continuation of past work [2,6,8,12, and 21] aimed at optimizing the creation of DNA nanostructures, but it also represents a new beginning. We have begun to consider the problem of optimizing structures that must obey a new set of constraints that more accurately reflect the present state of research in the field of DNA nanotechnology. It may be that in the future we will be able to specify something about the structure or size of all the possible nanostructures that can be built from a certain number of tiles. We wish to develop a working proof for the absolute minimum number of tiles required for cycles of all sizes in the octet truss.

It is true that there is still some flex to any arm that can presently be made, so that the assumption that arms are rigid is not absolutely sound. This is probably more of a problem for very large structures than it is for small structures, such as those we consider above. The flex of a structure is also related to the characteristics of the structure. These factors will constitute a good base for further study and new findings in DNA nanostructures in the octet truss.

The problem formulation is a significant part of our research, as it is with any mathematical project. It gives future researchers a comprehensible way to look

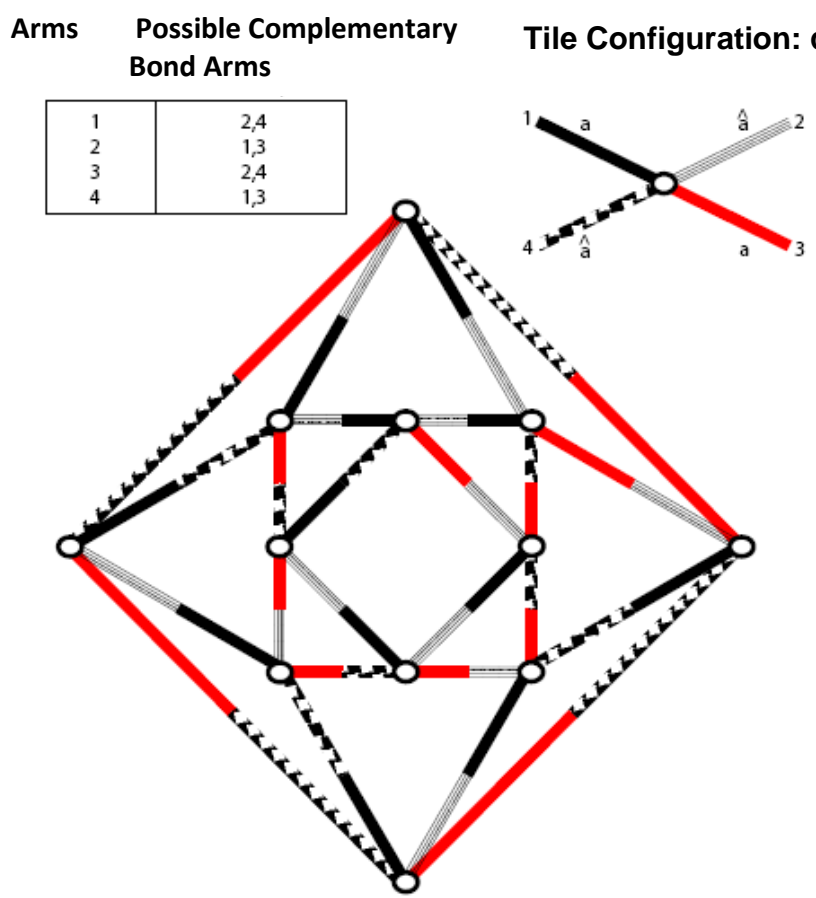

Figure 17. This diagram illustrates the construction part of the proof for the cuboctahedron. 
Arm Configurations Tile configuration: $\alpha_{1} \beta_{1} \gamma_{3}$
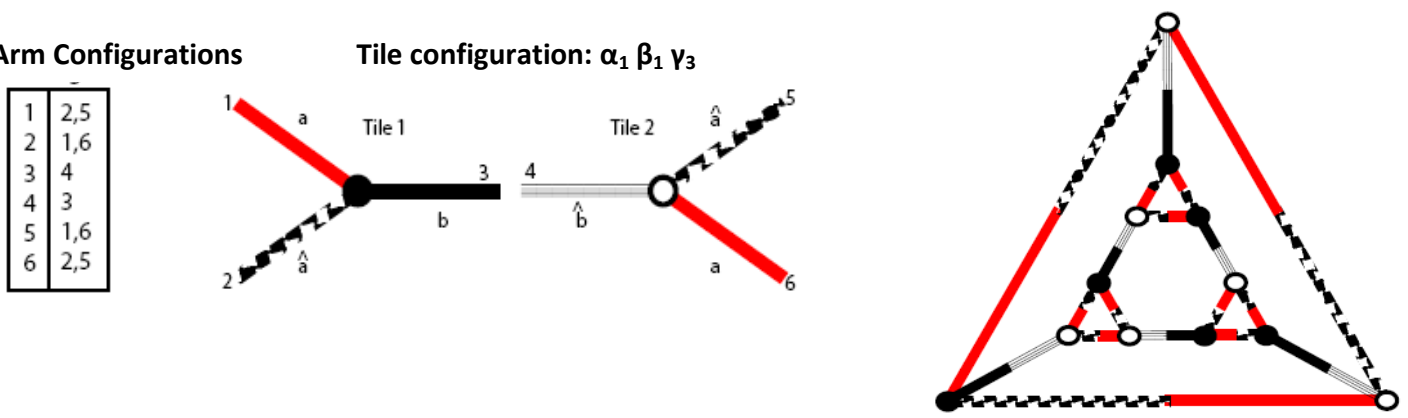

Figure 18. This diagram illustrates the construction part of the proof for the truncated tetrahedron.
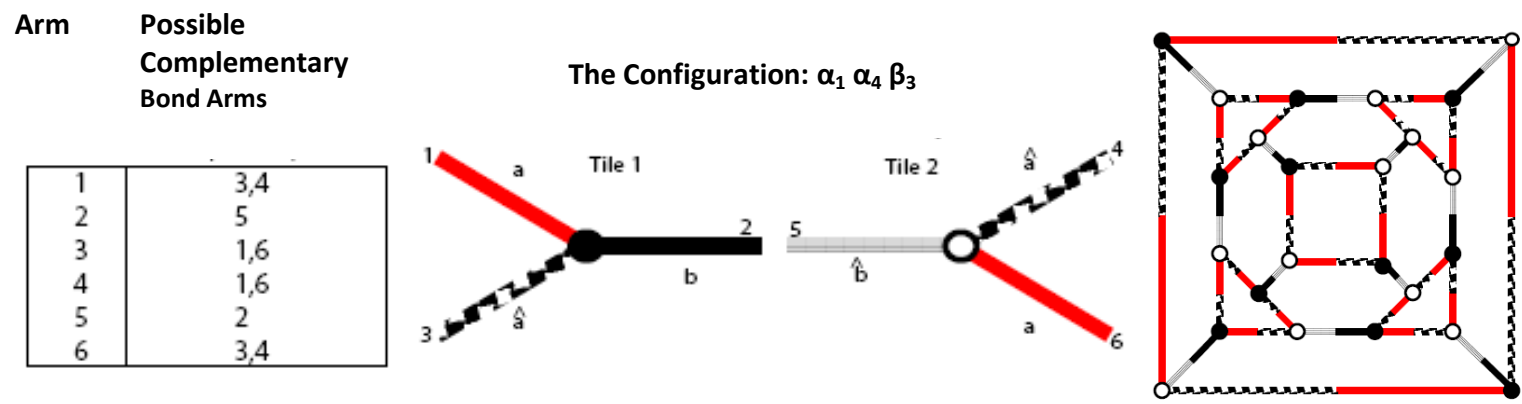

Figure 19. An illustration of the construction part of the truncated octahedron proof.

at the work we have done, and possibly continue it. As with our topic, DNA nanostructures, there are many definitions, theorems, and much background information in order to fully grasp the concepts of our work. We ultimately want to present our mathematical work in a manner that the researcher, who uses our findings, can understand. There are many possible paths to take with the information we have discovered. We can look at the tiles possible with more than four arms, and see what complexes can be made from these. It could be combined with computer simulations to implement a program check on our theories. This would make it more user-friendly for a person to experiment with the research we have completed. All of these possibilities, and more, need a foundation, which is the problem formulation and background information provided in this paper.

\section{ACKNOWLEDGEMNTS}

We thank Nadrian Seeman of Seemans Labs for his expertise in construction methods and design parameters; Natasha Jonoska from for fruitful discussion of design strategies; and our research advisors, Joanna Ellis-Monaghan and Greta Pangborn.

\section{REFERNCES}

1. L.M. Adelman, "Molecular Computation of Solutions to Combinatorial Proteins," Science 266 (1994) 1021-1024.

2. D. Archdeacon, "A survey of Self-Dual Polyhedra," Journal of Discrete Mathematics 51 (1992) 5-12.

3. L. Beaudin, J. Ellis Monaghan, D. Miller, and G. Pangborn, "Minimal Tile and Bond-Edge Types for Self-Assembling DNA Graphs," preprint July 2009.

4. J. Chen and N.C. Seeman, "Synthesis from DNA of a Molecule with the Connectivity of a Cube," Nature $\mathbf{3 5 0}$ (1991) 631-633.

5. F.A. Cotton, Chemical Applications of Group Theory $3^{\text {rd }}$ Edn. (John Wiley \& Sons, 1990) 
6. M. Cox. J. Ellis-Monaghan, T. Hughes, and K. Mondanaro, "Carbon Knots in the Octet Truss," The Journal of Mathematical Chemistry 43 (2008) 874891.

7. H. Dietz, S. Douglas, and W. Shih, "Folding DNA into Twisted and Curved Nanoscale Shapes," Science 329 (2009) 725-730.

8. J. Ellis-Monaghan, Transition Polynomials, Double Covers, and Bimolecular Computing," Congresses Numerantium 166 (2004) 181-192.

9. K. Freedman, J. Lee, Y. Li, d. Luo, V. Sbokeleva, and P. Ke, "Diffusion of Single Star-Branched Dendrimer-like DNA," Journal of Phys. Chem. B 109 (2005) 9839-9842.

10. R.B. Fuller, U.S. Patent 2986241 (1961).

11. Joseph A. Gallian, Contemporary Abstract Algebra $3^{\text {rd }}$ Edn. (Houghton Mifflin Company, 2006).

12. Y. He, T. Ye, M. Su, C. Zhang, A. Ribbe, W. Jiang, and C. Mao. "Hierarchical Self-Assembly of DNA into Symmetric Supermolecular Polyhedra," Nature 452 (2008) 198-202.

13. N. Jonoska, G. McColm, and A. Staninska, "Expectation and Variance of Self-Assembled Graph Structures," Springer LNCS 3892 (2006) 144-157. Note: in DNA Computing (DNA 12).

14. N. Jonoska, G. McColm, and A. Staninska, "Spectrum of a Pot for DNA Complexes," Springer LNCS 4287 (2006) 83-94. Note: in DNA Computing 12.

15. S.H. Ko, H. Liu, Y. Chen, and C. Mao, "DNA Nanotubes as Combinatorial Vehicles for Cellular Delivery,"
Biomacromolecules 9 (2008) 30393043.

16. T.H. LaBean and H. Li, "Constructing Novel Materials with DNA," Nano Today 2 (2007) 26-35.

17. D. Luo, "The Road from Biology to Materials," Materials Today 6 (2003) 3843.

18. John H. Reif (Editor), Proceedings of the Third Conference on Foundations of Nanoscience: Self-Assembled Architectures and Devices (FNANO06), Snowbird, Utah, (April 23-27, 2006), Published by Sciencetechnica (2006).

19. N. Seeman, "Nanotechnology and the Double Helix," Scientific American 209 (2004) 64-75.

20. N. Seeman, "An Overview of DNA Structural Nanotechnology," Molecular Biotechnology 37 (2007) 246-257.

21. W.M. Shih, J.D. Quispe, and G.F. Joyce, " A 1.7 Kilobase Single-Stranded DNA that folds into a Nanoscale Octahedron," Nature 427 (2004) 618621.

22. J.D. Watson and F.H.C. Crick, "A Structure for Deoxyribose Nucleic Acid," Nature 177 (1953) 737-738.

23. H. Yan, S.H. Park, G. Finklestein, J. Reif, and T. LaBean, "DNA-Templated Self-Assembly of Protein Arrays and Highly Conductive Nanowires," Science 301 (2003) 1882-1884.

24. Y. Zhang and N.C. Seeman, "Construction of a DNA-Truncated Octahedron," Journal of American Chemistry 116 (1994) 1661-1669.

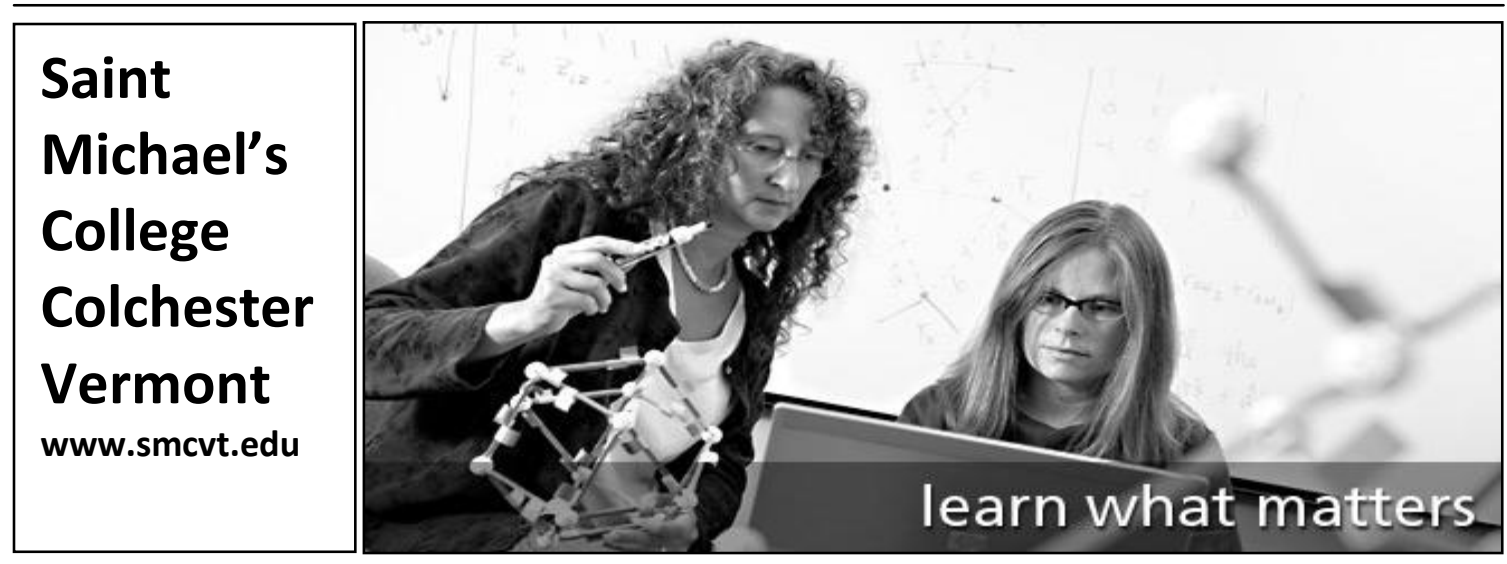




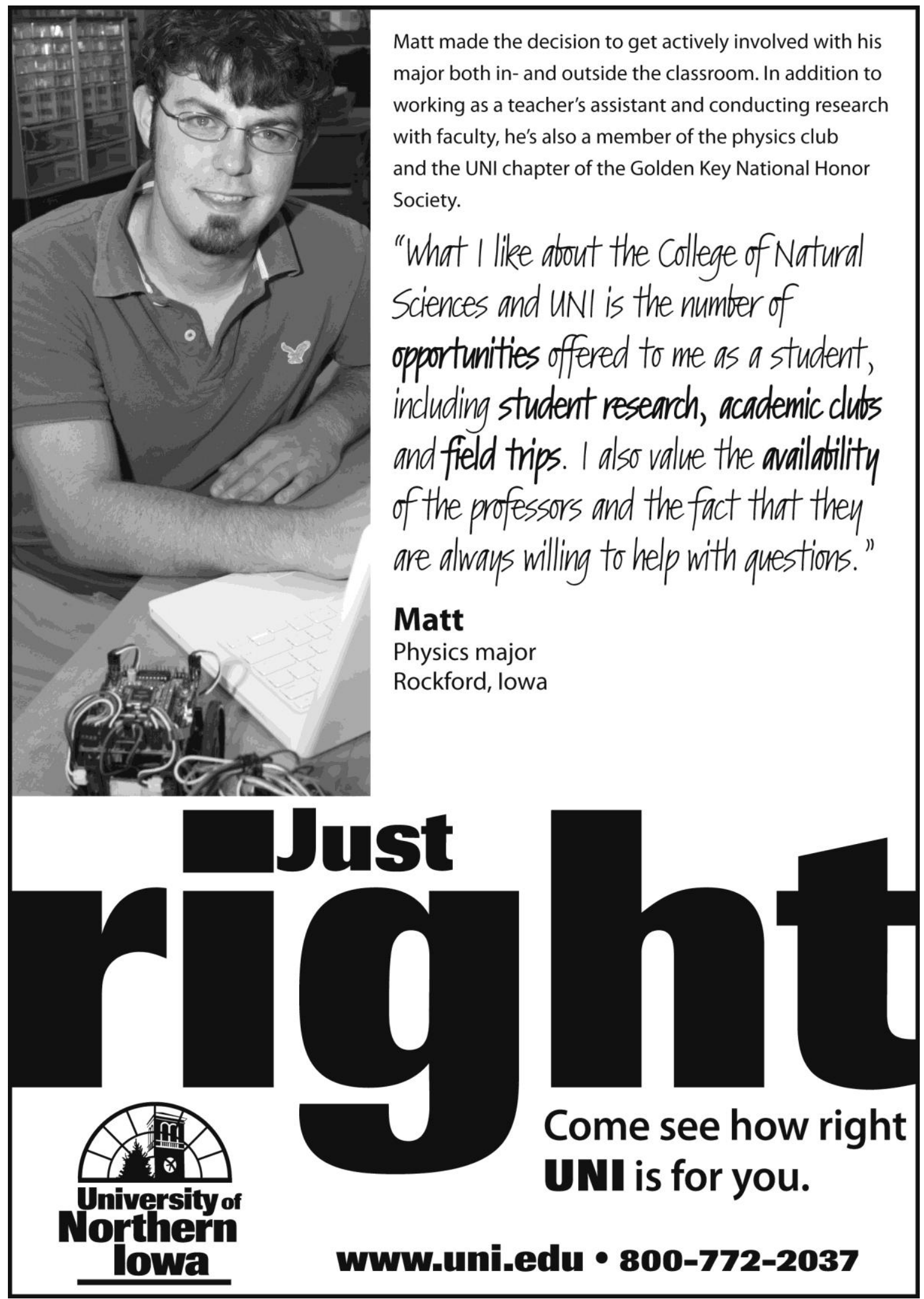




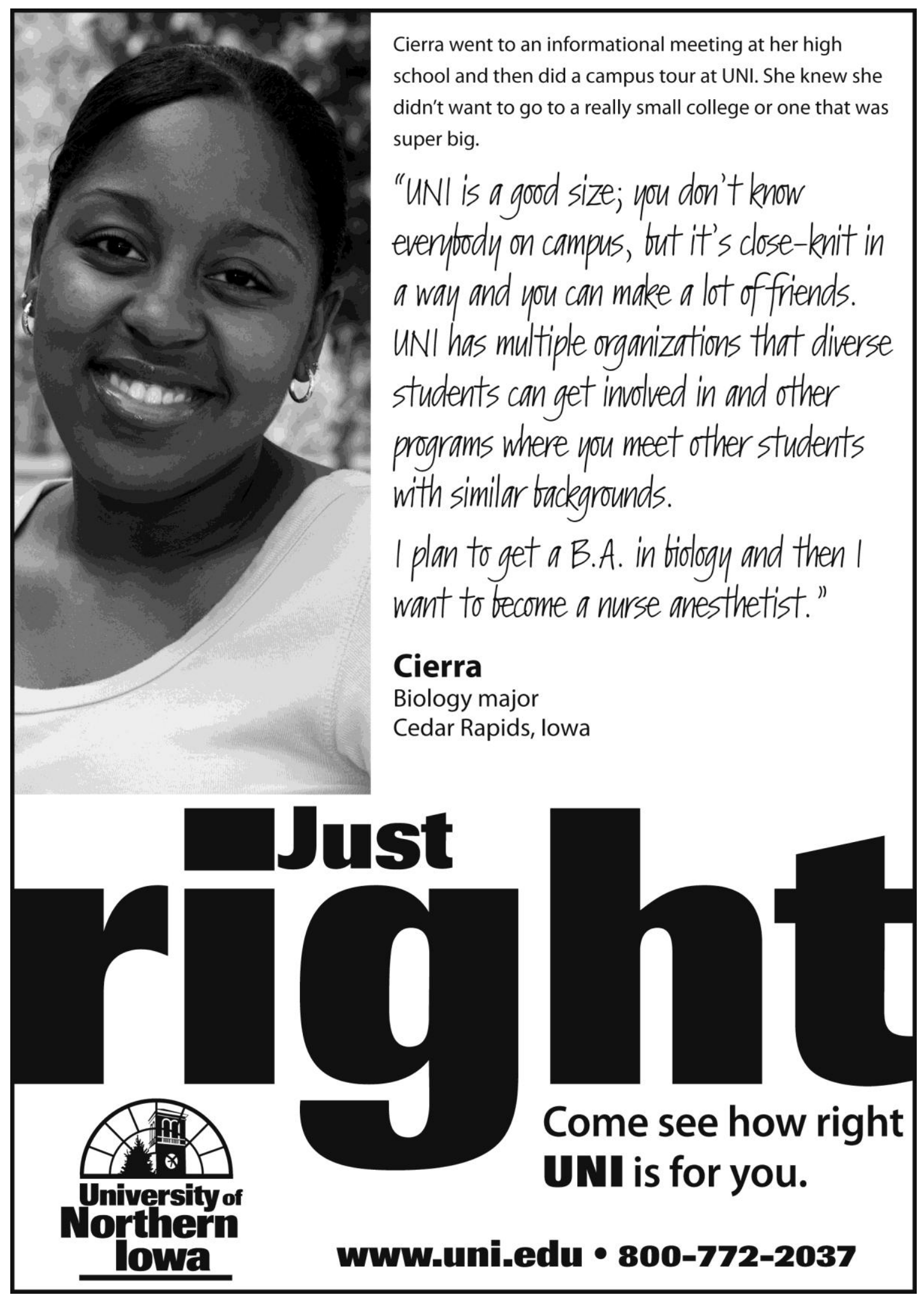

